\title{
Neutron Diffraction Study of Low-Cycle Fatigue Behavior in an Austenitic-Ferritic Stainless Steel
}

\author{
Ming-Wei Zhu' ${ }^{1} \cdot \mathrm{Nan} \mathrm{Jia}^{2} \cdot \mathrm{Feng} \mathrm{Shi}^{3} \cdot$ Bjørn Clausen $^{4}$
}

Received: 13 February 2015/Revised: 20 July 2015/Published online: 14 October 2015

(C) The Chinese Society for Metals and Springer-Verlag Berlin Heidelberg 2015

\begin{abstract}
By performing in situ neutron diffraction experiments on an austenitic-ferritic stainless steel subjected to lowcycle fatigue loading, the deformation heterogeneity of the material at microscopic level has been revealed. Based on the in situ neutron diffraction data collected from a single specimen together with the mechanical properties learned from the ex situ micro-hardness, a correlation has been found. The performance versus diffraction-profile correlation agrees with the cyclic-deformation-induced dislocation evolution characterized by ex situ TEM observation. Moreover, based on the refined neutron diffraction-profile data, evident strain anisotropy is found in the austenite. The high anisotropy in this phase is induced by the increase in dislocation density and hence contributes to the hardening of the steel at the first 10 cycles. Beyond 10 fatigue cycles, the annihilation and the rearrangement of the dislocations in both austenitic and ferritic phases softens the plastically deformed specimen. The study suggests that the evolution of strain anisotropy among the differently oriented grains and micro-strain induced by lattice distortion in the respective phases mostly affect the cyclic-deformationinduced mechanical behavior of the steel at different stages of fatigue cycles. The stress discrepancy between phases is not the dominant mechanism for the deformation of the steel.
\end{abstract}

KEY WORDS: Duplex steel; Neutron diffraction; Fatigue; Plastic deformation; Microstructure

\section{Introduction}

The duplex stainless steel used in the current study is an austenitic-ferritic steel possessing excellent mechanical properties and high resistance to stress corrosion cracking.

Available online at http://link.springer.com/journal/40195

Nan Jia

jian@atm.neu.edu.cn

1 School of Materials Science and Engineering, Shenyang Aerospace University, Shenyang 110136, China

2 Key Laboratory for Anisotropy and Texture of Materials (ATM), Northeastern University, Shenyang 110819, China

3 School of Science, Northeastern University, Shenyang 110819, China

4 Los Alamos National Laboratory, Los Alamos Neutron Science Center, Los Alamos, NM 87545, USA
Nowadays such duplex steels have widespread applications under various environments. Accordingly, a comprehensive study of the fatigue damage mechanisms is very important to develop and evaluate key engineering components of the steels for both future design and daily applications.

With modern experimental techniques and analytical tools, it is possible to estimate the stress to which a component is subjected in service. However, this is not sufficient for understanding the performance of the component, since in many cases an unexpected failure would occur because of the presence of internal stresses. For duplex steels, internal stresses could develop due to its different thermal expansions, yield stresses and stiffness among not only the different phases but also the differently oriented grains [1-3]. In recent years, the Bragg diffraction of synchrotron X-rays and neutrons in the crystal structure of materials has been extensively 
applied to study the micro-mechanical behaviors in engineering components $[4,5]$ because of their highpenetration ability for greater gauge volumes. The grains that diffract either X-ray or neutrons in the diffraction measurement are those with one specified lattice plane normal in a given direction. Therefore, this selective nature of diffraction technique allows the measurements of lattice strains subjected to different series of grain subsets along various crystallographic orientations. It also allows one to separate the elastic and plastic responses of the different phases. By far the fatigue properties of structural steels and superalloys have been widely investigated [6-12]. However, most of the work is focused on the correlation between the macro-mechanical behavior and microstructure. The information on the development of microscopic strain and load sharing among grains and between constituent phases during cyclic loading as well as their influence on the fatigue behavior of materials is still unclear [13-17]. In the investigation on fatigue of a $316 \mathrm{~L}$ austenitic stainless steel, Mineur et al. [6] found that texture influenced the stress-strain response and crack density of the material. Fatigue processes of dual-phase materials have also been studied, showing that the residual micro-stresses balanced between phases faded during fatigue $[15,16]$. For a duplex stainless steel consisting of austenite and ferrite, Johansson et al. [17] pointed out that the increasing residual micro-stress in the austenitic phase was induced by the rapid hardening of the phase, mainly controlling the cyclic loading responses of the material. Based on the aforementioned research achievements, we can conclude that the microscopic strain evolution and load partitioning within duplex steels have a significant influence on the fatigue behavior of the materials. Hence, it is necessary to consider various micro-mechanical interactions within steels for fatigue-behavior analyses. In the present work, based on time-of-flight (TOF) neutron diffraction experiments, the evolutions of strain/stress heterogeneity in both of austenitic and ferritic phases under cyclic loading were simultaneously characterized. A concurrent transmission electron microscopy (TEM) experiment was conducted separately to compare the microstructure rearrangements subjected to different numbers of fatigue cycles. Based on the analysis of elastic and plastic anisotropy of the constituent phases and microstructure changes of different fatigue cycles, the cyclic hardening and softening of the duplex steel are elucidated in detail. The results demonstrate that the evolution of strain anisotropy among the differently oriented grains and microstrain that is related to lattice distortion in the respective phases greatly affect the cyclic response of the austenitic-ferritic steel at different stages of fatigue.

\section{Material and Experimental Procedures}

The material investigated in the present work is a commercial austenite-ferrite $(\gamma-\alpha)$ duplex stainless steel, SAF 2507, provided by Sandvik AB. The as-received hot-rolled bar of $20 \mathrm{~mm}$ in diameter was supplied in solution annealed and water quenched condition. Our previous study shows that the microstructure consists of $58 \mathrm{vol} \%$ austenite and 42 vol\% ferrite and the average grain size is about $30 \mu \mathrm{m}$ in the both phases [5]. The nominal chemical composition (in weight percent) of the steel is presented in Table 1 . The $0.2 \%$ offset yield strength and fracture tensile ductility are $606 \mathrm{MPa}$ and $30 \%$, respectively. The ultimate strength is $830 \mathrm{MPa}$. A set of cylindrical specimens of $6 \mathrm{~mm}$ diameter and $44 \mathrm{~mm}$ gauge length were machined along the axis of the as-received bar material so that the loading direction was parallel to the rolling direction (RD) during the fatigue tests.

The in situ fatigue measurements were taken using the Spectrometer for Materials Research at Temperature and Stress (SMARTS) [18] at Los Alamos National Laboratory. The specimens were cycled under uniaxial tensilecompressive loading under total strain control with an amplitude of +1 and $-1 \%$, respectively. The cyclic loading frequency was $1.0 \mathrm{~Hz}$. The specimens were aligned $45^{\circ}$ to the incident neutron beam. The lattice planes with the normal direction perpendicular to the loading axis and the lattice planes with the normal direction parallel to the loading axis were measured by the two detectors banks that were fixed at $2 \theta$ of $-90^{\circ}$ and $90^{\circ}$, respectively. A schematic presentation of the experimental arrangement is shown in Fig. 1. The direction of incident neutron beam is marked with a blue arrow. Relative to the specimen alignment, there are two detector banks to collect the diffracted neutron of the gauged volume in two orthogonal directions. Since the specimen is aligned along the cyclic loading direction, we define the detection bank collecting the diffracted neutrons from the grains, orienting to the loading direction, is in the loading direction. Likewise, the transverse-direction detector, orthogonal to the loading direction, collects the diffracted neutrons from the grains oriented in the transverse direction.

Considering that the TOF is proportional to the neutron wavelength, i.e.,

Table 1 Chemical composition of the duplex stainless steel (in wt \%)

\begin{tabular}{llllllllll}
\hline $\mathrm{C}$ & $\mathrm{Si}$ & $\mathrm{Mn}$ & $\mathrm{P}$ & $\mathrm{S}$ & $\mathrm{Cr}$ & $\mathrm{Ni}$ & $\mathrm{Mo}$ & $\mathrm{N}$ & $\mathrm{Fe}$ \\
\hline 0.03 & 0.8 & 1.2 & 0.035 & 0.02 & 25.0 & 7.0 & 4.0 & 0.3 & Bal. \\
\hline
\end{tabular}




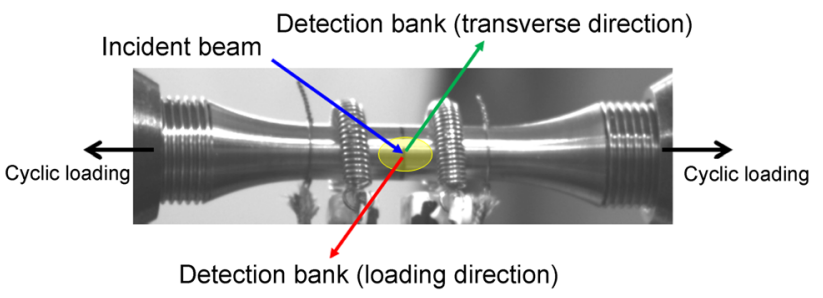

Fig. 1 Schematic presentation to show the arrangement of the in situ neutron diffraction measurements

$\lambda=h t /(m L)$,

where $h$ is Planck's constant, $m$ is the neutron mass and $L$ is the traveling path of neutrons, we obtain the interplanar spacing, $d_{h k l}$, of the hkl plane in terms of Bragg's law, i.e.,

$d_{h k l}=\lambda_{h k l} /\left(2 \sin \theta_{h k l}\right)=h t_{h k l} /\left(2 m L \sin \theta_{h k l}\right)$,

where $t_{h k l}$ is the TOF for the $h k l$ plane, which was determined by fitting individual peak in the diffraction spectra by the GSAS software [19]. For the investigated duplex steel, the $\{111\} \gamma,\{200\} \gamma,\{220\} \gamma$ and $\{311\} \gamma$ reflections of austenite and the $\{110\} \alpha,\{200\} \alpha$ and $\{211\} \alpha$ reflections of ferrite were analyzed. Then, the lattice strain, $\varepsilon_{h k l}$, was calculated as

$\varepsilon_{h k l}=\left(d_{h k l}-d_{0}\right) / d_{h k l}=\left(t_{h k l}-t_{0}\right) / t_{h k l}$,

where $d_{0}$ and $t_{0}$ denote the interplanar spacing and the neutron TOF for the specified hkl plane of a stress-free material, respectively. In the current work, the measurement at the initial load of $5 \mathrm{MPa}$ was taken as the stressfree state of the specimen, which means that phase stresses due to thermal mismatch between austenite and ferrite are not considered in the current analysis. The diffraction data were collected for the loading points located at the maximum strain of 1 and $-1 \%$, respectively, at different fatigue cycles: 1 st, 5th, 10th, 50th, 75th, 225th and 500th cycles.

Ex situ micro-hardness tests on the austenitic and ferritic phases of the duplex steel after different fatigue cycles were measured using a $401 \mathrm{MVD}^{\mathrm{TM}}$ digital sclerometer with an applied load of $0.49 \mathrm{~N}$ for $15 \mathrm{~s}$. The separately micro-hardness measurements are important to compare the bulk mechanical properties side by side with the above in situ neutron diffraction experiments and the following ex situ TEM tests. A diamond indentor of standard geometry, typically a $136^{\circ}$ square-based pyramidal diamond, was used, and the average diagonal length of the indents was approximate $30 \mu \mathrm{m}$ in the both phases. At least 10 measurements at different gauge volumes (each volume consists of several adjacent grains of the same phase) were taken to obtain an averaged micro-hardness value for each individual phase under the different deformation conditions. Then, to investigate the deformation-induced microstructure of the steel, disks with a diameter of $3 \mathrm{~mm}$ were taken from several ex situ fatigue-deformed specimens subjected to different cyclic numbers for the ex situ TEM investigation. The TEM specimens prepared from the as-received material, without any cyclic deformation, are the reference. A Tecnai G220 transmission electron microscope operated at $200 \mathrm{kV}$ was used. The investigated sample surfaces that were parallel to the loading axis were mechanically polished to $30 \mu \mathrm{m}$ and then electro-polished in a solution of $10 \mathrm{vol} \% \mathrm{HClO}_{4}$ and $90 \mathrm{vol} \% \mathrm{C}_{2} \mathrm{H}_{5} \mathrm{OH}$ at $0{ }^{\circ} \mathrm{C}$ with the plateau current of $50 \mathrm{~mA}$. To remove the oxide formed on the surface, ion polishing was then carried out using an argon ion gun AG-21, with ion beam parameters of $3 \mathrm{keV}$ and $10 \mu \mathrm{A}$.

\section{Results and Discussion}

\subsection{Low-Cycle Fatigue Experiments}

Figure 2 shows the cyclic stress-strain behavior of the austenitic-ferritic steel with the applied strain amplitude of $\varepsilon=1 \%$ (black squares) and $-1 \%$ (red triangles), respectively. Note that the low-cycle fatigue experiments were under a strain-controlled mode. With the strain-controlled fatigue loading, the same $1 \%$ strain yielded greater stresses upon cyclic loading within the tenth fatigue cycle. Similarly, under $-1 \%$ strain control, the specimen demanded greater and greater compression as a function of fatigue cycle up to about the tenth fatigue cycle. The observed phenomena are so-called the cyclic hardening. The cyclic hardening of the studied steel transited to cyclic softening after the tenth fatigue cycle. It is evident that the continuous decrease in the absolute values of the stresses occurs at the both +1 and $-1 \%$ macroscopic strains beyond the tenth cycle. It can be seen that within the possible

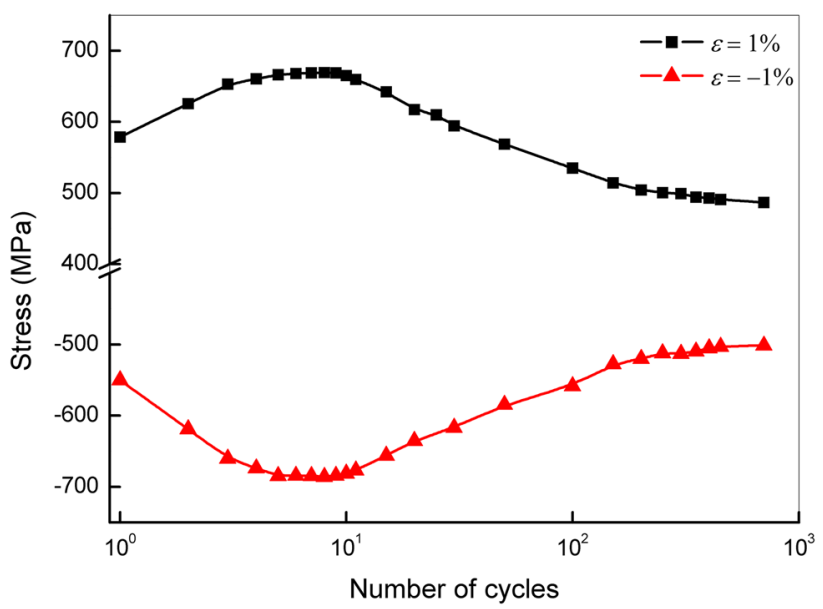

Fig. 2 Stress amplitude as a function of number of loading cycles for the austenitic-ferritic steel 
magnitude of the applied strain range [7], a well-defined cyclic hardening-softening transition during fatigue cycles is observed in the investigated steel.

\subsection{In situ Neutron Diffraction Results}

Since the macroscopic stress evolution subjected to tension and compression is symmetric, we assume that there is no Bauschinger effect at the bulk level. On the other hand, besides the averaged-bulk-stress evolutions shown in Fig. 2, we select the neutron data, subjected to $1 \%$ strain, as a function of fatigue cycles to reveal the lattice rearrangements in different orientations and of different phases first. The neutron diffraction revealed lattice strains along the loading direction for different reflections, $\varepsilon_{h k l}$, are plotted as a function of the fatigue cycles in Fig. 3. Due to the nature of the neutron cross-section area of the Fe atoms, which composed most of the investigated stainless steel, the diffraction data are with sufficient intensity yielding the errors as low as $1 \%$. The error bars are marked all over the neutron data. Because of the levels of the errors are too small, those error bars are even smaller than the sizes of the legends in Fig. 3. The advantage of the SMARTS is its orthogonal detector banks as depicted in Fig. 1. With two orthogonal detector banks, the neutrons diffracted by the grains oriented to both loading and transverse direction were collected simultaneously. Figure $3 a$ and $b$ reveals the lattice strain evolution of the $\gamma$ phase in two orthogonal directions, respectively. Similarly, the evolutions of the $\alpha$ phase in loading and transverse directions are shown in Fig. 3c, d, respectively.

It can be seen that for most of the lattice planes in the respective phases, the strain increases with increasing load cycles until about the tenth cycle, after which the value decreases and levels out at a stable level. This trend of the lattice strain evolution indicates that the intergranular strain, i.e., the maximum difference in strain among the orientated grains, $\left|\varepsilon^{\max }-\varepsilon^{\min }\right|$, may develop in the both phases during the cyclic deformation. Relatively strong strain anisotropy is found in the austenite due to the higher work-hardening rate of this phase. As for the lattice strain distribution among different $\{\mathrm{hkl}\}$ planes in the duplex
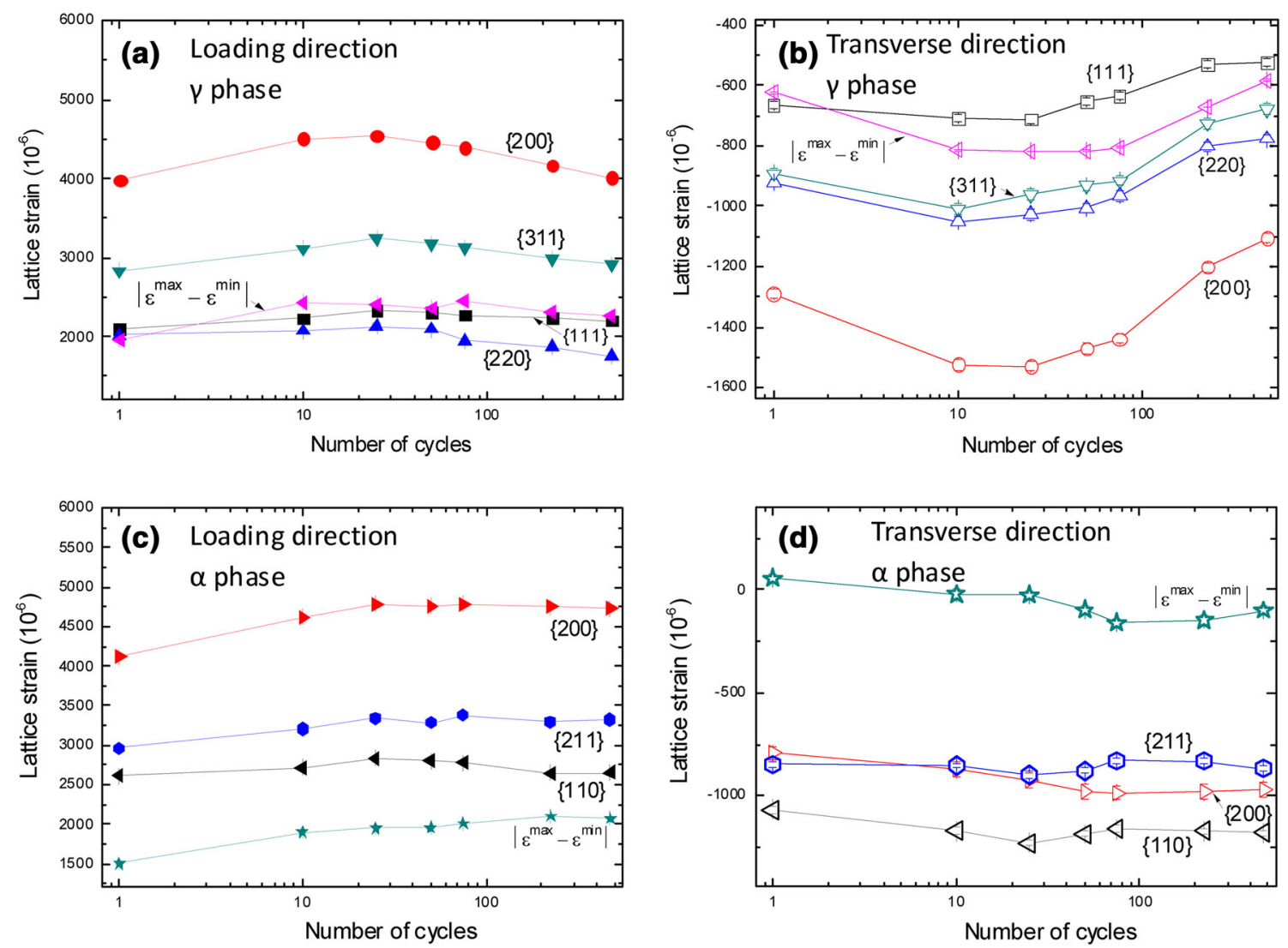

Fig. 3 Responses of lattice strains to the fatigue cycles along the loading direction and the transverse direction for different reflections in austenite $(\gamma)$ and ferrite $(\alpha)$ (the duplex steel is at the macro-strain of $1 \%$; $\left|\varepsilon^{\mathrm{max}}-\varepsilon^{\mathrm{min}}\right|$ denotes the intergranular strain among the differently orientated grains in each phase): a $\gamma$ phase, loading direction; $\mathbf{b} \gamma$ phase, loading direction; $\mathbf{c} \alpha$ phase, loading direction; $\mathbf{d} \alpha$ phase, loading direction 
steel, it can be found in our previous investigation where elastic and plastic anisotropic responses of lattice strains to the applied stress is identified in each phase of the same material under tensile loading [20]. Similarly, there are opposite trends in the transverse direction. However, the lattice strain evolutions of the $\gamma$ phase in both loading and transverse directions (Fig. 3a, b) are more proportional to the bulk cyclic hardening and softening transition (Fig. 2) than that of the $\alpha$ phase (Fig. 3c, d).

To explore micro-strains that are associated with the lattice imperfections within the steel, the neutron diffraction peak widths for the specimens after different fatigue cycles are characterized by the full width at half maximum (FWHM) for the Gaussian part of the peak profile function, as shown in Fig. 4. Figure $4 a$ and $b$ is the peak-width evolution of the $\gamma$ phase, in loading and transverse directions, respectively. Similarly, the evolutions of the FWHM for the $\alpha$ phase in loading and transverse directions are shown in Fig. $4 \mathrm{c}$ and $\mathrm{d}$, respectively. The unit of the FWHM peak width is in milliseconds of time of flight. Obviously, the general trends of the evolution of peak width are similar for the differently oriented grains. As obtained from the lattice reflections in the both phases, some diffraction peaks are broadened within the first 10 cycles and then slightly narrowed after the 50th cycle. This trend is more significant for the austenite $\{220\}$ and $\{311\}$ reflections as well as the ferrite $\{110\}$ and $\{200\}$ reflections, compared to other lattice planes. That is to say, at around the tenth cycle micro-strain possesses the maximum value compared to the other load cycles. This feature is in accordance with the stress evolution as shown in Fig. 2. Based on the recent work [21, 22], grain refinement, microstrain, stacking faults, twin boundaries, and any other imperfections within a microstructure yield the diffraction line broadening. It should be noted that, in the respective phases, the difference in lattice strains among the constituent lattice planes is an indication of plastic anisotropy (Fig. 3). This lattice strain anisotropy arising from the grain-to-grain interaction also leads to the activation of geometrically necessary dislocations, so as to accommodate the gradients of plastic deformation within the steel. Conversely, the dislocations may cause distinct shift of the different hkl reflections, additionally leading to the lattice strain anisotropy [23]. Therefore, in this study it is inferred that the accommodation of incompatibility within the individual grains and among the different grains jointly contributes to the changes of the peak width in terms of FWHM. This compatibility finally leads to the stress
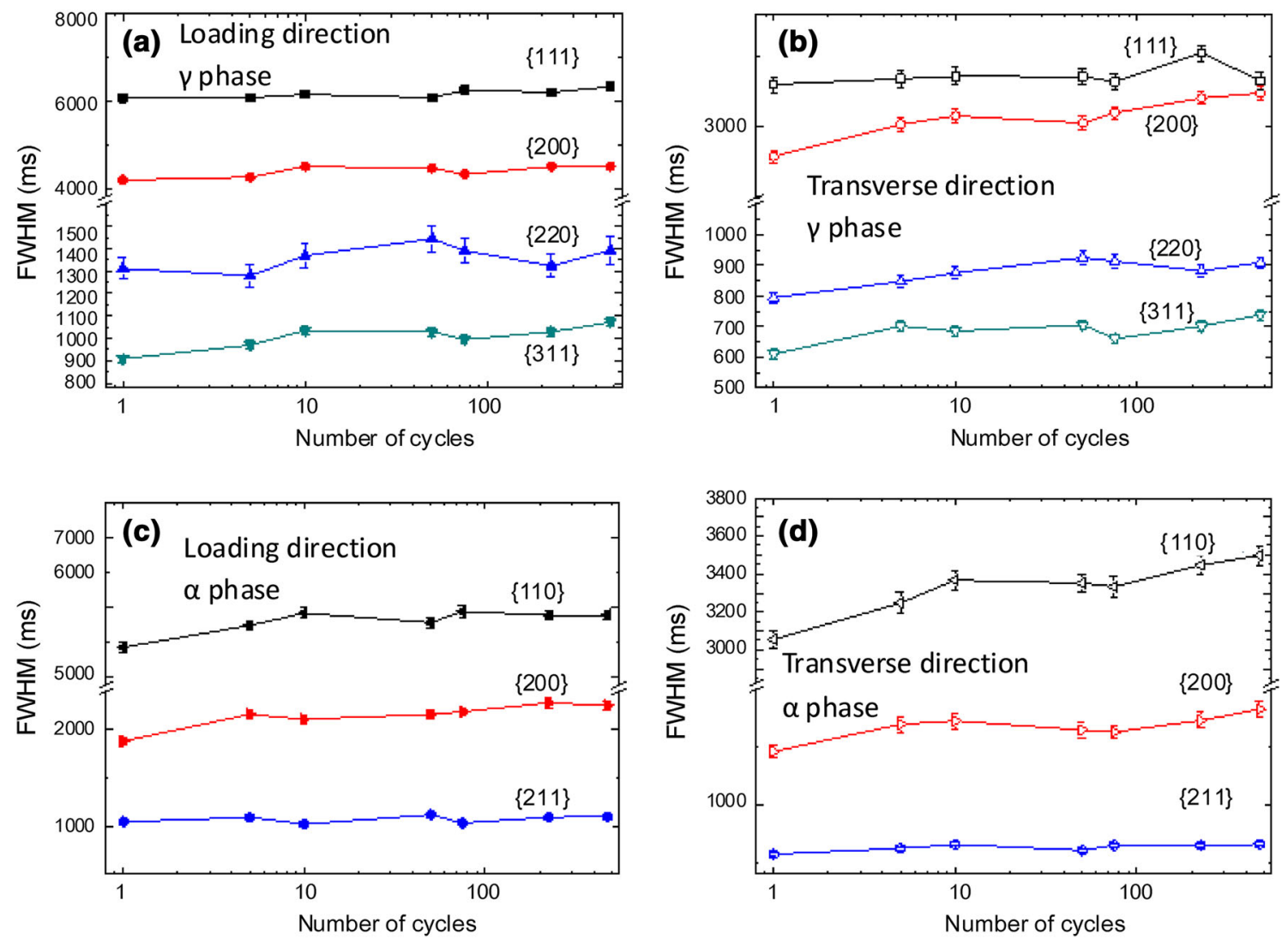

Fig. 4 Responses of diffraction peak widths to the fatigue cycles for different reflections in austenite $(\gamma)$ and ferrite $(\alpha)$ : a $\gamma$ phase, loading direction; $\mathbf{b} \gamma$ phase, loading direction; $\mathbf{c} \alpha$ phase, loading direction; $\mathbf{d} \alpha$ phase, loading direction 
gradients and stress concentrations not only within single crystals but also close to the phase boundaries. The inhomogeneous distribution of stress is also sensitive to substructures of the steel during fatigue, which will be discussed later.

As for the duplex steel during deformation, the stress distribution is heterogeneous due to not only the grain orientations but also the variation of phases. Based on the current diffraction experiments, the stress tensor components of each diffracting grain family could be determined from the three measured principal strain tensor components, $\varepsilon_{11}, \varepsilon_{22}$ and $\varepsilon_{33}$ (11 denotes the RD, 22 and 33 denote the two orthogonal directions normal to the RD, and $\varepsilon_{22}=\varepsilon_{33}$ ) according to the isotropic model using Hooke's law. Namely, the stress is calculated by

$\begin{aligned} \sigma_{i i} & =E\left[\varepsilon_{i i}+v\left(\varepsilon_{11}+\varepsilon_{22}+\varepsilon_{33}\right) /(1-2 v)\right] /(1+v), i \\ & =1,2,3\end{aligned}$

where $E$ is the diffraction elastic constants and $v$ is Poisson's ratio in the subset of grains with their $\{\mathrm{hkl}\}$ crystal planes normal to the scattering vector. All the parameters determined by the Kröner model [24] for the austenite and the ferrite are listed in Table 2. Considering that $\{311\}$ reflection in austenite and $\{211\}$ reflection in ferrite best represent the average stress of the respective phases [25], in the current isotropic model, calculation is made merely using the lattice strains measured on those reflections for the phases. Figure 5 shows the stress of each individual phase as a function of the applied load cycle of the duplex steel based on the isotropic model. As can be seen, the stress discrepancy between phases jumps to about $150 \mathrm{MPa}$ during the first cycle with the macro-strain varying from 0 to $1 \%$. This prominent increase in phase-to-phase interaction is due to the mechanical mismatch between phases, i.e., the much higher elastic module of the ferrite compared to that of the austenite [26, 27]. Before loading up to the tenth cycle, the difference in stress remains between the phases and only a minor increase in the stress discrepancy could be identified with the elevated fatigue cycles. Moreover, it is seen that the austenitic phase undertakes less load than the ferritic phase, indicative of the relatively high work hardening of the austenite compared with that of the ferrite during this period. After 10 cycles which approximately correspond to the onset of the softening of

Table 2 Diffraction elastic constants $(E)$ and Poisson's ratio $(v)$ in the subset of grains with their reflection plane $\{h k l\}$ normal to the scattering vector, determined by the Kröner model for austenite $(\gamma)$ and ferrite $(\alpha)$

\begin{tabular}{lccccc}
\hline & $\{200\} \gamma$ & $\{200\} \alpha$ & $\{220\} \gamma$ & $\{211\} \alpha$ & $\{311\} \gamma$ \\
\hline$E(\mathrm{GPa})$ & 152 & 181 & 211 & 228 & 184 \\
$\nu$ & 0.33 & 0.321 & 0.264 & 0.275 & 0.294 \\
\hline
\end{tabular}

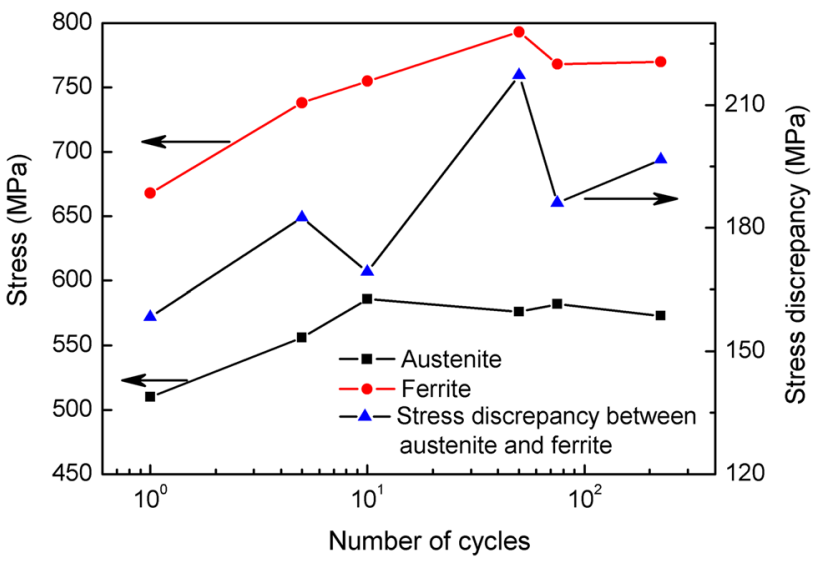

Fig. 5 Isotropic model calculated stress of the constituent phases and stress discrepancy between phases in the duplex steel as a function of number of fatigue cycles

the steel, the austenite shows a decrease in stress. Accordingly, this leads to a significant increase in the phase-to-phase interaction with increasing applied cycles. Afterward, the stress discrepancy between phases starts to decrease once again, which may be attributed to the subsequent softening of the ferrite. Then, the intrinsic elastoplastic properties of the constituent phases explain the fluctuant but prominent stress partition between the phases. Further work is still required for quantitatively explaining the interactions of compatibility stresses in such a twophase material under cyclic loading by developing some advanced numerical or analytical models, such as a dedicated multiphase crystal plasticity model [20] capable of handling an anisotropic matrix. This is, however, beyond the scope of the current paper.

\subsection{Micro-Hardness Tests}

The micro-hardness versus fatigue cycle curves for the austenitic and ferritic phases are shown in Fig. 6, in which the average micro-hardness for the duplex steel is also indicated. For the austenite, an abrupt rise of hardness is observed at the initial cycles and an obvious peak, located at the tenth cycle, appears in the curve of the hardness versus cycle. This process corresponds to the fast work hardening of the austenitic phase and is followed by a decrease in the micro-hardness with increasing fatigue cycle. When comparing the stress distribution between phases that is obtained by the micro-hardness tests and the isotropic model (Fig. 5), some deviation is found. This is ascribed to the major contribution of the grain-orientationdependent stress to the phase-to-phase interaction. It should also be mentioned that the high work-hardening rate of the deformed metallic phase explains why lattice strains (as characterized by the change of diffraction peak position) 


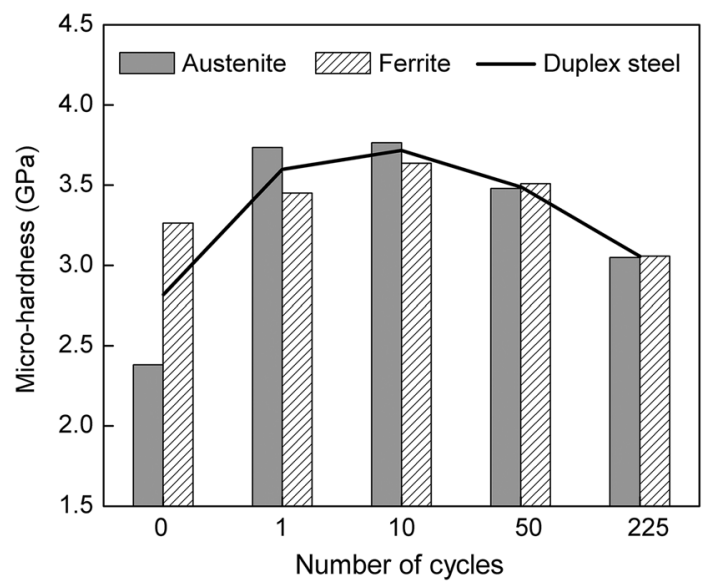

Fig. 6 Responses of micro-hardness to the fatigue cycles for the constituent phases and the duplex steel

and micro-strains (as characterized by the broadening of diffraction peak width) in austenite increase with increasing load cycle at the first 10 cycles. Compared with the austenitic phase, the peak hardness for the ferritic phase still appears at the tenth cycle, but is weakened in magnitude, in the curve of the hardness versus cycle number. With the further elevated cycle numbers, the micro-hardness of the ferrite decreases to a low level comparable to the value when the steel is at the as-received state. During fatigue, the reduction in hardness from the tenth cycle to the 225th cycle is evident for the both phases, indicating that the applied load has been taken up more uniformly in the steel with cyclic deformation.

\subsection{TEM Observation of the Deformation Microstructures}

TEM photographs show microstructures in the as-received duplex steel and in the specimens fatigued with different load cycles. By characterizing a great many areas for each specimen, it is known that the microstructures shown below are representative of the features of the steel at different stages of deformation. As can be seen in Fig. 7, the asreceived steel exhibits a low dislocation density in both austenitic and ferric phases. However, after fatigue tests, the TEM photographs of the fine microstructures that evolve with deformation clearly show the development of dislocation structures in the both phases, as presented in Figs. 8, 9 and 10. After the first cycle, pileups of planar dislocations and accumulation of dislocation arrays are observed in the austenitic grains (Fig. 8a). Consistent experimental results could be found in some other's work on typical face-centered-cubic (fcc) materials with low stacking fault energy [28]. Meanwhile, the substructure containing stacking faults that appear as parallel fringe patterns between two partial dislocations is observed (Fig. 8b, c), which means that a

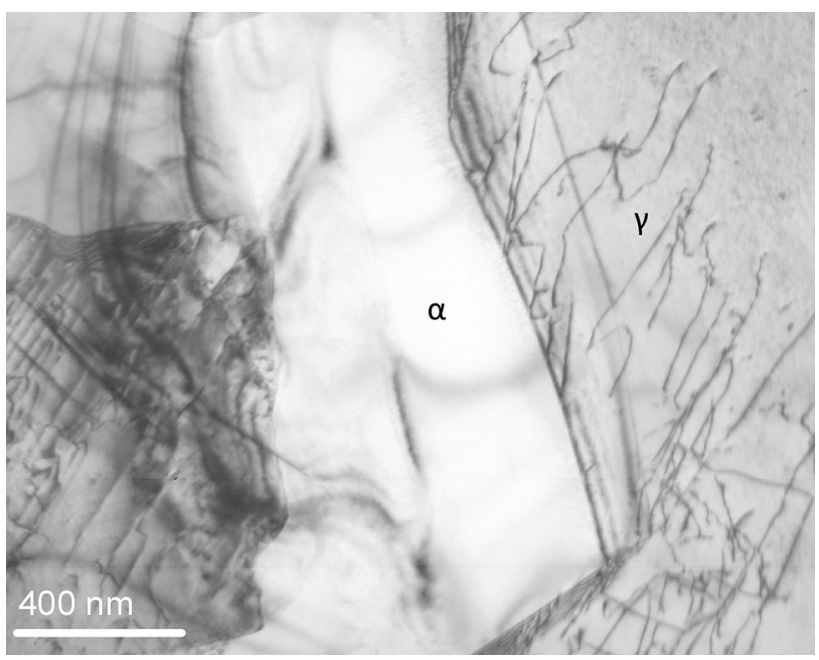

Fig. 7 TEM image of the as-received duplex steel showing low dislocation density in both austenitic and ferritic phases

particular configuration of dislocations, i.e., the LomerCottrell dislocation [29], may be formed and act as a barrier to the motion of other dislocations. However, in the interior of ferritic grains, only a little bit increase in dislocation density could be identified at this stage of deformation (Fig. 8d). After the tenth cycle, the austenitic grains generally develop a denser distribution of planar arrangement of dislocations along the $\{111\}$ glide plane, and some loop-like dislocations are also randomly distributed in this phase (Fig. 9a, b). A theoretical study showed that the presence of $\mathrm{N}$ in austenite can lower its stacking fault energy [30]. And it is also found that short-range ordering may be induced by the affinity between $\mathrm{N}$ and $\mathrm{Cr}$ in this phase [31]. Therefore, in the current experiment, cross-slip is hindered and planar slip is promoted because of these effects, which thereby increases the strain-hardening rate of the austenitic phase. In the ferrite, the dislocation configuration with bowed segments and small loops is observed. Namely, the microstructure in the ferritic grains after 10 cycles starts to be dominated by screw dislocations (Fig. 9c). Several researchers $[32,33]$ have reported this type of microstructure for ferrite in duplex stainless steels. It should be mentioned that, for the both phases at this stage of deformation, denser dislocations are observed to congregate at grain boundaries and fewer dislocations are distributed at grain interiors. Moreover, a dense dislocation structure is also identified at some phase boundaries (Fig. 9d). The dislocation pileups at some interfaces may cause the inhomogeneous distribution of internal stress within the steel. To this stage, for the austenite the source of the diffraction peakwidth broadening comes from its denser dislocations and dislocation walls, and for the ferrite the peak-width broadening is attributed to the randomly distributed dislocations within the phase. 

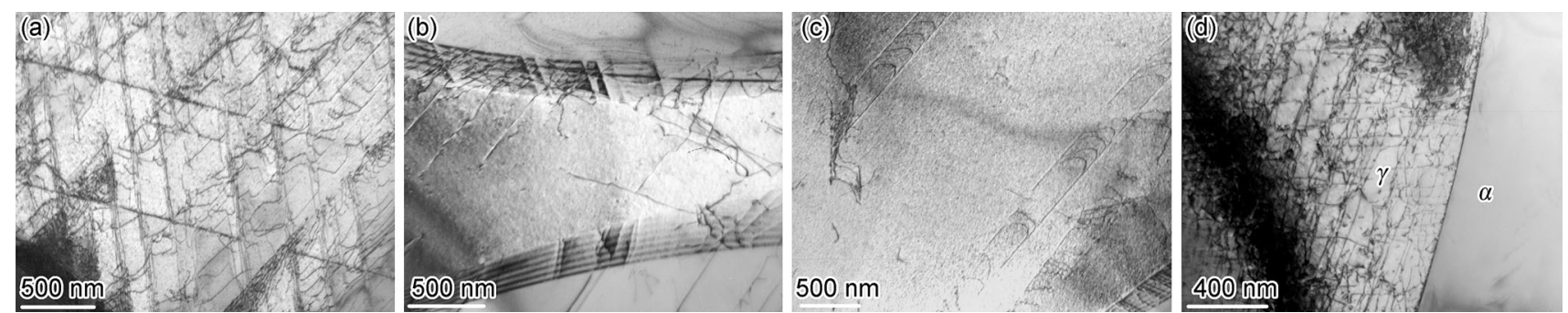

Fig. 8 Microstructures of the duplex steel after the first cycle of fatigue tests: a pileups of planar dislocations and accumulation of dislocation arrays in austenitic grains; $\mathbf{b}$, $\mathbf{c}$ substructure containing stacking faults that appear as parallel fringe patterns between two partial dislocations in austenite; $\mathbf{d}$ only a slight increase in dislocation density is identified in the interior of ferritic grains
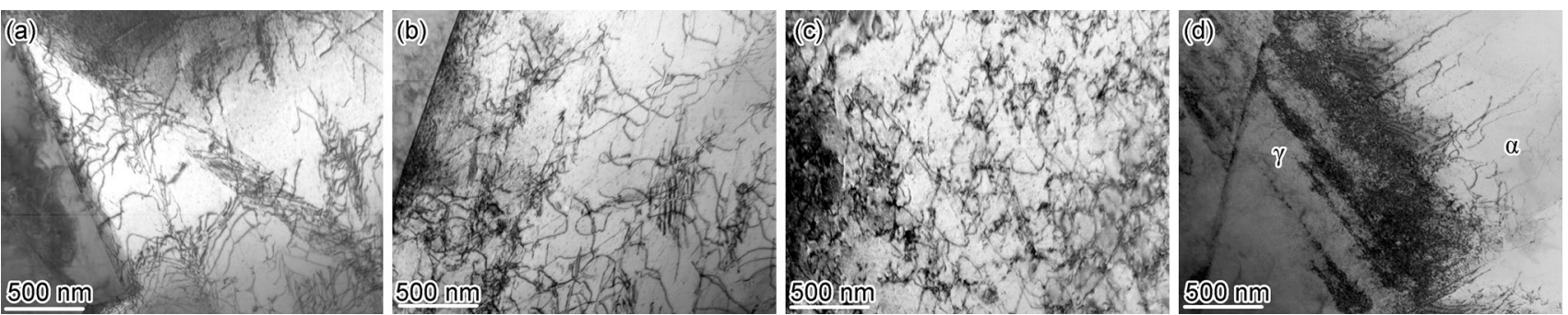

Fig. 9 Microstructures of the duplex steel after the tenth cycle of fatigue tests: $\mathbf{a}, \mathbf{b}$ a denser distribution of planar arrangement of dislocations and some loop-like dislocations in the austenite; $\mathbf{c}$ ferrite is dominated by screw dislocations; $\mathbf{d}$ dense dislocations are identified at some phase boundaries
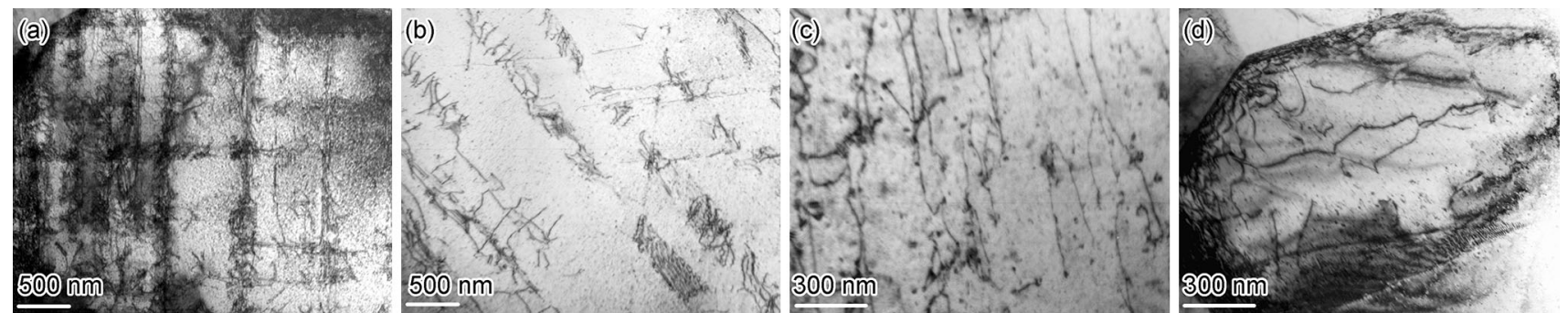

Fig. 10 Microstructures of the duplex steel after the 225th cycle of fatigue tests: $\mathbf{a}, \mathbf{b}$ the hierarchical dislocation structure is formed in austenite; $\mathbf{c}$ the dislocation density in ferrite is lowered; $\mathbf{d}$ in some ferritic grains, a cellular dislocation structure is formed and a much lower dislocation density is observed at the cell interiors than the cell boundaries

For the specimen after the 225th cycle, the hierarchical dislocation structure with some dislocations accumulated at the dislocation walls and some randomly distributed between the walls is identified in the austenite (Fig. 10a, b). Although some dislocation debris is still visible between the walls, the dislocation density has been significantly reduced. Similar dislocation structure has been previously reported in a fcc nickel-based alloy during uniaxial tensile loading [23]. One may note that a slight increase in diffraction peak width can be identified in the austenitic grains after approximate 75 cycles (Fig. 4a, b). This may be attributed to the decreasing crystallite size within a blocky structure showing typical dimensions of $<0.5 \mu \mathrm{m}$ (see Fig. 10a). Similar size changes of the dislocation-cell structure could also be found in other nickel-based alloys [34]. On the other hand, the morphology of stacking faults has been changed, showing that the planar arrays are composed of randomly oriented dislocation segments. This type of array may reduce the critical resolved stress that is required for dislocation 
glide within the Lomer-Cottrell dislocation configuration, thus impairing work-hardening behavior and facilitating strain accommodation of the austenitic phase during deformation. For the ferrite, a lower dislocation density is comparable to the smaller peak-width data refined from the diffraction peak profiles of the earlier fatigue cycles. In some ferritic grains, a cellular dislocation structure emerges and a much lower dislocation density is observed at the cell interiors than the cell boundaries. To this stage, by means of neutron diffraction, TEM, together with the conventional mechanical tests, the above agreement among TEM, neutron diffraction and hardness tests conclude that the causes of hardening and softening in the austenitic-ferritic steel during fatigue depend on the dislocation structure evolution. Because of the different elastic and plastic properties of the constituent phases, the stress discrepancy between phases is found at the maximum macro-strain of $1 \%$ after the various fatigue cycles. However, since the effect of the higher elastic modulus and yield stress of the ferrite is compensated by that of the higher work-hardening rate of the austenite, there is no obvious development of the phase-to-phase interaction with increasing cycle number. Accordingly, for the hardening of the steel during the first 10 cycles and the softening with the greater cycles, it is attributed to the evolution of strain anisotropy among the differently oriented grains as well as micro-strain that is related to the lattice distortion in the respective phases. Another recent lattice strain asymmetry subjected to fatigue cycles also shows that the development of the strain anisotropy is caused by cyclic loading [35]. These can be supported by the TEM investigations, where the development of dislocation structures with increasing applied cycles is observed agreeable with the micro-mechanical behavior of the individual phases. It should be mentioned that the relatively high work-hardening rate of the austenitic phase leads to the higher intergranular strain in this phase, which plays a major role in the hardening response of the steel before the tenth cycle. For the softening of the steel, however, it is contributed by the evolution of dislocation structures as well as the decreasing dislocation density in the both austenitic and ferritic phases.

To our knowledge, the results discussed above represent so far the first detailed experimental investigations of the deformation heterogeneity at microscopic level of an austenitic-ferritic steel during fatigue, which has also been verified by the evolution of microstructures of the steel at different stages of loading. The study thus provides fundamental experimental input for future micro-mechanical modeling aiming at the evaluation and prediction of the mechanical performance of multiphase materials under cyclic loading.

\section{Summary}

Neutron diffraction, micro-hardness test and TEM observation have been performed to study the micro-mechanical behavior of an austenitic-ferritic duplex steel under fatigue loading. The main results can be summarized as follows:

1. The cyclic hardening and softening of the steel are attributed to the evolution of strain anisotropy among the differently oriented grains and micro-strain that is related to the lattice distortion in the respective phases.

2. Even if prominent phase-to-phase interaction has been found at the maximum macro-strain in each loading cycle, the stress discrepancy between phases shows a weak relationship with the cyclic response of the steel.

3. The higher strain anisotropy of the austenite which is caused by an increasing dislocation density in this phase mainly contributes to the hardening of the steel. The evolution of dislocation structures as well as the decreasing dislocation density in the both austenitic and ferritic phases contributes to the softening of the steel.

Acknowledgments We gratefully acknowledge the financial support of the Fundamental Research Funds for the Central Universities (Nos. N130510001 and L1502029), the Program for New Century Excellent Talents in University (No. NCET-13-0104) and the National Natural Science Foundation of China (Nos. 51202256, 51201027). We also gratefully acknowledge E-Wen Huang at National Central University for very valuable discussions. The Lujan Neutron Scattering Center at the Los Alamos Neutron Science Center is funded by the Department of Energy's Office of Basic Energy Science. The Los Alamos National Laboratory is operated by the Los Alamos National Security LLC under the DOE Contract of DEAC52-06NA25396.

\section{References}

[1] S. Harjo, Y. Tomota, Acta Mater. 47, 353 (1998)

[2] Y. Tomota, P. Lukas, S. Harjo, J.H. Park, N. Tsuchida, D. Neov, Acta Mater. 51, 819 (2003)

[3] C. Larsson, B. Clausen, T.M. Holden, M.A.M. Bourke, Scr. Mater. 51, 571 (2004)

[4] Y. Tomota, H. Tokuda, Y. Adachi, M. Wakita, N. Minakawa, A. Moriai, Y. Morii, Acta Mater. 52, 5737 (2004)

[5] N. Jia, R.L. Peng, Y.D. Wang, G.C. Chai, J. Johansson, G. Wang, P.K. Liaw, Acta Mater. 54, 3907 (2006)

[6] M. Mineur, P. Villechaise, J. Mendez, Mater. Sci. Eng. A 286, 257 (2000)

[7] I. Alvarez-Armas, M.C. Marinelli, S. Hereñú, S. Degallaix, A.F. Armas, Acta Mater. 54, 5041 (2006)

[8] S. Wroński, A. Baczmański, R. Dakhlaoui, C. Braham, K. Wierzbanowski, E.C. Oliver, Acta Mater. 55, 6219 (2007)

[9] X. Li, T.F. Jing, M.M. Lu, R. Xu, B.Y. Liang, J.W. Zhang, J. Mater. Sci. Technol. 27, 364 (2012)

[10] C.C. Shih, N.J. Ho, H.L. Huang, J. Mater. Sci. 45, 818 (2010)

[11] R.L. Peng, G.C. Chai, N. Jia, Y.D. Wang, S. Johansson, Fatigue Fract. Eng. Mater. Struct. 31, 892 (2008)

[12] S.M. Yin, S.X. Li, J. Mater. Sci. Technol. 29, 775 (2013) 
[13] Y.V. Taran, M.R. Daymond, J. Schreiber, Appl. Phys. A 74, $1385(2002)$

[14] T. Lorentzen, M.R. Daymond, B. Clausen, C.N. Tomé, Acta Mater. 50, 1627 (2002)

[15] J.D. Almer, J.B. Cohen, B. Moran, Mater. Sci. Eng. A 284, 268 (2000)

[16] I. Alvarez-Armas, U. Krupp, M. Balbi, S. Hereñú, M.C. Marinelli, H. Knobbe, Int. J. Fatigue 41, 95 (2012)

[17] J. Johansson, M. Odén, Metall. Mater. Trans. A 31, 1557 (2000)

[18] M.A.M. Bourke, D.C.E. Dunand, E. Üstündag, Appl. Phys. A 74, 1707 (2002)

[19] A.C. Larson, R.B. Von Dreele, Report No. LAUR 86-748, Los Alamos National Laboratory, Los Alamos, 2004

[20] N. Jia, R.L. Peng, D.W. Brown, B. Clausen, Y.D. Wang, Metall. Mater. Trans. A 39, 3134 (2008)

[21] T. Ungár, O. Castelnau, G. Ribárik, M. Drakopoulos, J.L. Béchade, T. Chauveau, A. Snigirev, I. Snigireva, C. Schroer, B. Bacroix, Acta Mater. 55, 1117 (2007)

[22] B. Jóni, E. Schafler, M. Zehetbauer, G. Tichy, T. Ungár, Acta Mater. 61, 632 (2013)

[23] E.W. Huang, R. Barabash, N. Jia, Y.D. Wang, G.E. Ice, B. Clausen, J. Horton, P.K. Liaw, Metall. Mater. Tran. A 39, 3079 (2008)

[24] E. Kröner, Z. Phys. 151, 504 (1958)
[25] B. Clausen, T. Lorentzen, T. Leffers, Acta Mater. 46, 3087 (1998)

[26] G. Simmons, H. Wang, Single Crystal Elastic Constants and Calculated Aggregate Properties: A Handbook, 2nd edn. (MIT, Cambridge, 1971)

[27] H.M. Ledbetter, Phys. Status Solidi A 85, 89 (1984)

[28] T.P. Kruml, J. Polak, J. Obrtlik, S. Degallaix, Acta Mater. 45, 5145 (1997)

[29] T.H. Courtney, Mechanical Behavior of Materials, 2nd edn. (McGraw-Hill, New York, 2000), p. 116

[30] G. Wahlberg, G.L. Dunlop, in Proceedings of Stainless Steels'87, Institute of Metals, London, pp. 291, 1987

[31] J.-O. Nilsson, Scr. Metall. 17, 593 (1983)

[32] J.J. Moverare, M. Odén, Mater. Sci. Eng. A 337, 25 (2002)

[33] I. Alvarez-Armas, P. Evrard, V. Aubin, S. Degallaix-Moreuil, Mater. Test. 51, 349 (2009)

[34] E.W. Huang, R.I. Barabash, Y.D. Wang, B. Clausen, L. Li, P.K. Liaw, G.E. Ice, Y. Ren, H. Choo, L.M. Pike, D.L. Klarstrom, Int. J. Plast. 24, 1440 (2008)

[35] E.W. Huang, R.I. Barabash, B. Clausen, Y.L. Liu, J.J. Kai, G.E. Ice, P. Woods, P.K. Liaw, Int. J. Plast. 26, 1124 (2010) 